\title{
THE SHAPE OF THE MOON
}

\author{
S. K. RUNCORN and S. HOFMANN \\ Department of Geophysics and Planetary Physics, School of Physics, \\ University of Newcastle upon Tyne, England
}

\begin{abstract}
The determination of the heights of points on the lunar surface by Earth based astronomy using the geometrical librations, although individually of low accuracy, still provides our best method of obtaining the global shape of the Moon. The intrinsic scatter of the results arises from the effects of 'seeing' and simple statistical analysis is required to derive valid conclusions about the shape. Baldwin's method of fitting ellipsoidal surfaces to the points on the maria and uplands, separately by the method of least squares proves to be a valuable tool.

Analyses of the ACIC points and of the Pic du Midi studies of G. A. Mills show that good first descriptions of the global shape of the Moon for both the maria and uplands are triaxial ellipsoids with their long axes within $10^{\circ}$ of the Earth direction, the major axis of the maria being about $1.3 \mathrm{~km}$ smaller than that of the uplands. Of particular significance is that the ellipticity of these surfaces is about $2 \frac{1}{2}$ times greater than the dynamical ellipticity; thus the non-hydrostatic figure of the Moon is not simply the result of distortion from a uniform Moon during its early history. The angular variation in density within the Moon cannot be simply a phenomena within the crust but must extend to a great depth. Convection could provide an explanation.

The departures of the lunar surface from the idealised ellipsoids are also of interest. The circular maria are systematically depressed relative to the maria ellipsoid: can the mascons have adjusted isostatically since their formation? Systematic differences in height between the western and eastern southern uplands are also noted.
\end{abstract}

\section{The Ellipticity of the Lunar Surface}

The global shape of the Moon, that is, its shape neglecting local topography, is perhaps its most fundamental property. Yet the external shape of the Moon has been a controversial issue for a century and the major questions at issue are as follows. Can the Moon's overall shape be described by 'an earthward bulge', i.e. is there a strong second harmonic in its external surface? Is the bulge really directed towards the mean position of the Earth? If so, what is the height of the bulge, i.e. the excess of the earthward radius over that in the plane of the sky.

Astronomers, using the method of geometrical librations to determine the heights of points on the lunar surface from the centre of the Moon's figure, found values between 0 and $20 \mathrm{~km}$ for the height of this bulge. A major step towards understanding the method was taken by Baldwin (1949) who analysed points on the maria and uplands separately; he showed both that the maria were systematically lower than the uplands and that the spheroid fitted to each set of points had nearly the same ellipticity. However Kopal (1967) and Goudas (1963) analysed the points as a whole, fitting them to a spherical harmonic series and showed that higher harmonics than the second, especially the fourth, were needed to describe the external surface adequately.Therefore they were inclined to regard 'the lunar bulge' as meaningless or at least an oversimplification of the description of the shape of the Moon. Runcorn (1967) however argued that because the maria surfaces were distinctly younger than the uplands and evidently of a different physiochemical origin, the surfaces associated with the Moon which are 
of true physical significance are the surfaces of the uplands and maria taken separately rather than the external surface of the Moon.

Particularly the natural step of comparing the external gravitational field of the Moon with its shape would be more revealing if the two regions of the surface, evidently of different chemical composition (and therefore probably of different densities) were separately considered. So Runcorn and Gray (1967) and Runcorn and Shrubsall (1968), following Baldwin's further analysis (1963), analysed the more numerous data of the ACIC program by methods similar to but rather more general than his. A new set of data has now been obtained by Mills (1968) and this is now in this paper subjected to the same analysis, details of which are given in Runcorn and Gray (1967).

The difficulty of what in principle is a simple problem arises because the scatter of the data exceeds the quantities to be determined. Each height determination is subject to errors arising from the astronomical phenomenon of seeing and from the varying lighting of the peaks and other lunar features studied which, unlike stars, are not point sources. Even the differential displacements of the points arising from the geometrical librations are about the same as the angular resolving power of the telescopes used, multiplied by the Earth-Moon distance. Thus the Rayleigh diffraction limit of earth based astronomy sets a limit to the accuracy of a height determination as emphasized by Kopal (1967). The shape of the Moon by the method of geometrical librations is thus a problem of seeing through noise but Runcorn (1967) showed that, providing the errors are random, a determination of the height of the bulge or the low harmonics of its shape should be possible using, as Baldwin did, about 100 points. The much more accurate methods now available through space technology to determine height do not have anywhere near the overall coverage of the earth-based observations, so that the analysis of the geometrical libration data is still most important. The more numerous data now available enable many aspects of the Moon's shape to be discussed which were not hitherto possible. The only way to ensure that the effects are not the result of systematic errors is to use and compare different sets of measurements. Table I compares the different sets of results obtained by previous workers and the present paper giving the length of the semi-axes $a_{x}, a_{y}$ and $a_{z}$ of the ellipsoids which best fit points on the maria and the uplands separately ( $z$ is the polar axis and $x$ the Earth aligned axis). Mills (1968) has listed lunar coordinates which we now analyse and compare with previous data. His data is divided, in Figure 1, into heights of points on the maria and for the uplands and the absolute heights of these lunar features are plotted against the sine of their angular distance $(K)$ from the centre of the lunar disc. This shows clearly that the mean level of the maria points is below that of the uplands and also shows the reality of the earthward bulge at the centre of the disc. The full lines show the heights of the best fitting ellipsoids for each set using Mills' data and the dotted lines those fitting ACIC data, using the analysis of Runcorn and Shrubsall (1968), the $a_{y}$ and $a_{z}$ axes being taken as equal. The two pairs of lines show the good agreement between the analyses of the two sets of data.

In Figure 2 the variation is shown in the standard deviation of the mean error with the angle of orientation in the final fit of the ellipsoids to the maria ard upland points, 


\section{TABLE I}

Axes of best fitting ellipsoids to uplands and maria comparing with previous papers

\begin{tabular}{|c|c|c|c|c|c|}
\hline & \multicolumn{5}{|c|}{ Uplands ellipsoid } \\
\hline & \multirow{2}{*}{$\begin{array}{l}\text { Runcorn } \\
\text { and Gray } \\
\operatorname{rot}^{n} \text { about } \\
z \text { axis }\end{array}$} & \multirow{2}{*}{$\begin{array}{l}\text { Runcorn and } \\
\text { Shrubsall } \\
\operatorname{rot}^{n} \text { about } \\
z \text { axis }\end{array}$} & \multirow[b]{2}{*}{$\begin{array}{l}\operatorname{rot}^{n} \text { about } \\
x \text { axis }\end{array}$} & \multicolumn{2}{|c|}{ Present paper } \\
\hline & & & & $\begin{array}{l}\operatorname{rot}^{n} \text { about } \\
z \text { axis }\end{array}$ & $\begin{array}{l}\operatorname{rot}^{n} \text { about } \\
x \text { axis }\end{array}$ \\
\hline No. of points & 90 & 581 & 581 & 542 & 542 \\
\hline & & $a_{x}^{\prime}$ & $a_{x}^{\prime \prime}$ & $a_{x}^{\prime}$ & $a_{x}^{\prime \prime}$ \\
\hline$a_{x}($ in $\mathrm{km})$ & 1739.63 & 1739.91 & 1739.92 & 1740.19 & 1740.17 \\
\hline$a_{y}($ in $\mathrm{km})$ & 1736.65 & 1736.59 & 1736.30 & 1737.31 & 1736.97 \\
\hline$a_{z}($ in $\mathrm{km})$ & 1736.15 & 1736.90 & 1737.27 & 1736.48 & 1736.20 \\
\hline$a_{x}-a_{y}($ in $\mathrm{km})$ & 2.98 & 3.32 & 3.62 & 2.88 & 3.20 \\
\hline$a_{x}-\left(a_{z}+a_{y}\right) / 2$ & 3.23 & 3.165 & 3.14 & 3.30 & 3.58 \\
\hline $\begin{array}{l}\text { Angle between } a^{\prime} x \text { of } \\
\text { best fit and axis } \\
\text { towards Earth }\end{array}$ & $0^{\circ}$ & $7^{\circ} \mathrm{W}$ & - & $5^{\circ} \mathrm{E}$ & \\
\hline $\begin{array}{l}\text { Angle between } a^{\prime \prime}{ }_{z} \text { of } \\
\text { best fit and polar axis }\end{array}$ & - & - & $40^{\circ} \mathrm{W}$ & - & $32.5^{\circ} \mathrm{W}$ \\
\hline$a_{z}^{\prime \prime}-a_{y}^{\prime \prime}$ & - & - & 0.97 & - & -0.77 \\
\hline
\end{tabular}

Maria ellipsoid

\begin{tabular}{lrrrrr} 
No. of points & \multicolumn{1}{c}{106} & 391 & 391 & 385 & \multicolumn{1}{c}{385} \\
$a_{x}$ (in km) & 1738.99 & 1738.62 & 1738.56 & 1739.05 & 1739.02 \\
$a_{y}($ in $\mathrm{km})$ & 1735.40 & 1735.28 & 1735.67 & 1735.97 & 1736.13 \\
$a_{z}$ (in km) & 1734.56 & 1735.44 & 1735.42 & 1734.66 & 1734.68 \\
$a_{x}-a_{y}($ in km) & 3.59 & 3.34 & 2.89 & 3.08 & 2.89 \\
$a_{x}-\left(a_{z}+a_{y}\right) / 2$ & 4.01 & 3.26 & 3.02 & 3.73 & 3.61 \\
$\begin{array}{l}\text { Angle between } a^{\prime}{ }_{x} \text { of } \\
\text { best fit and axis }\end{array}$ & $5^{\circ} \mathrm{E}$ & $5^{\circ} \mathrm{E}$ & - & $5^{\circ} \mathrm{E}$ & - \\
towards Earth & & & & & \\
$\begin{array}{l}\text { Angle between } a^{\prime \prime}{ }_{z} \text { of } \\
\text { best fit and polar axis }\end{array}$ & - & - & $30^{\circ} \mathrm{W}$ & - & $5^{\circ} \mathrm{W}$ \\
$a^{\prime \prime}{ }_{z}-a^{\prime \prime}{ }_{y}$ & - & - & & & -1.45 \\
\end{tabular}

Uplands axis minus maria axes

$$
\begin{aligned}
& a_{x}(\text { in } \mathrm{km}) \\
& a_{y}(\text { in } \mathrm{km}) \\
& a_{z}(\text { in } \mathrm{km}) \\
& a_{x}-a_{y}(\text { in } \mathrm{km}) \\
& a_{x}-\left(a_{z}+a_{y}\right) / 2
\end{aligned}
$$

Angle between $a^{\prime \prime}{ }_{z}$ of best fit and polar axis $a_{z}^{\prime \prime}-a^{\prime \prime} y$

$\begin{array}{ll}0.64 & 1.29 \\ 1.25 & 1.31 \\ 1.59 & 1.46\end{array}$

Fit by limb

Goudas (1963) $35^{\circ} \mathrm{W}$

1.36
0.63
1.85

1.14

1.34

1.82

1.15

0.84

1.52
Fit by limb

Watts (1963) $35^{\circ} \mathrm{W}$

1.12 


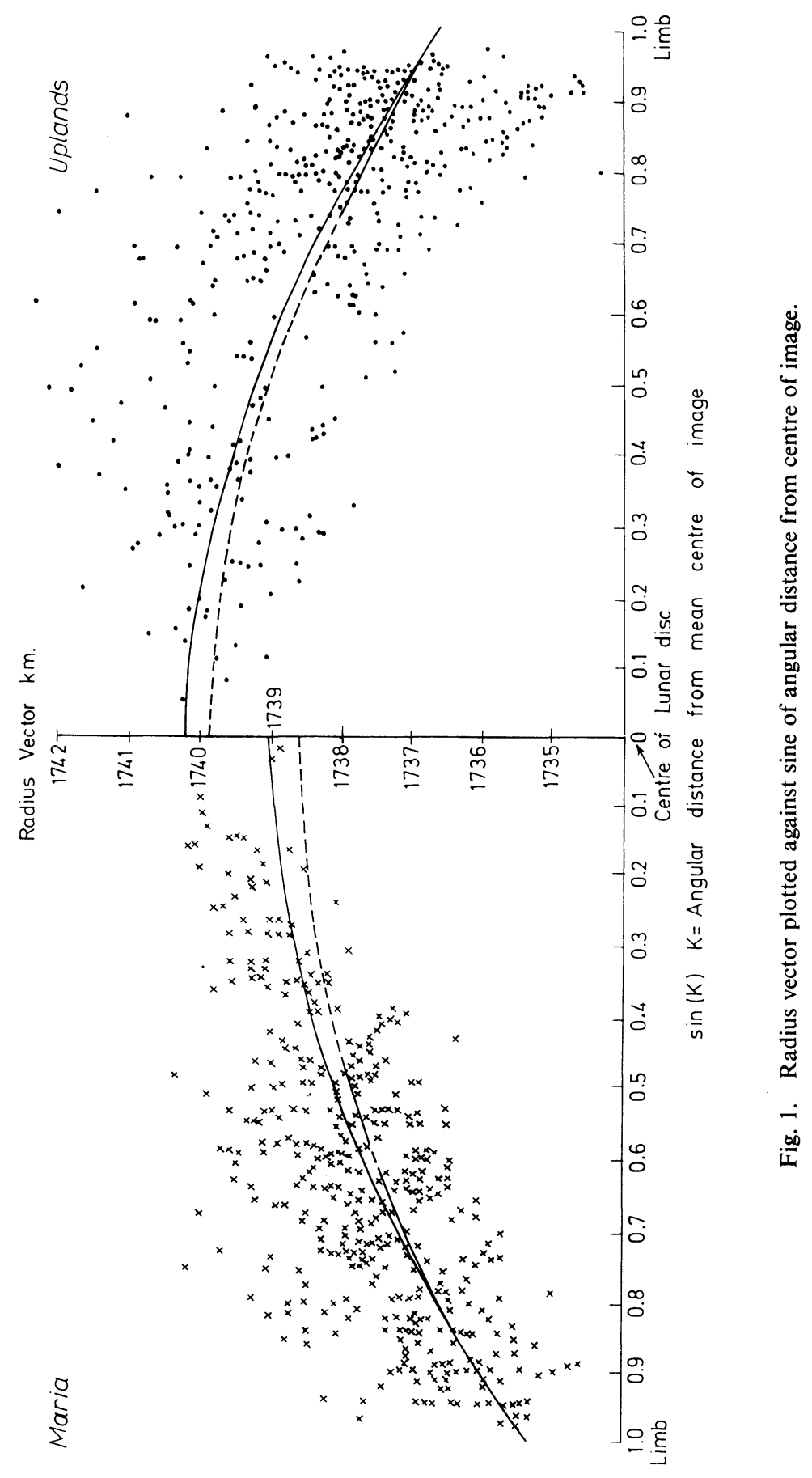



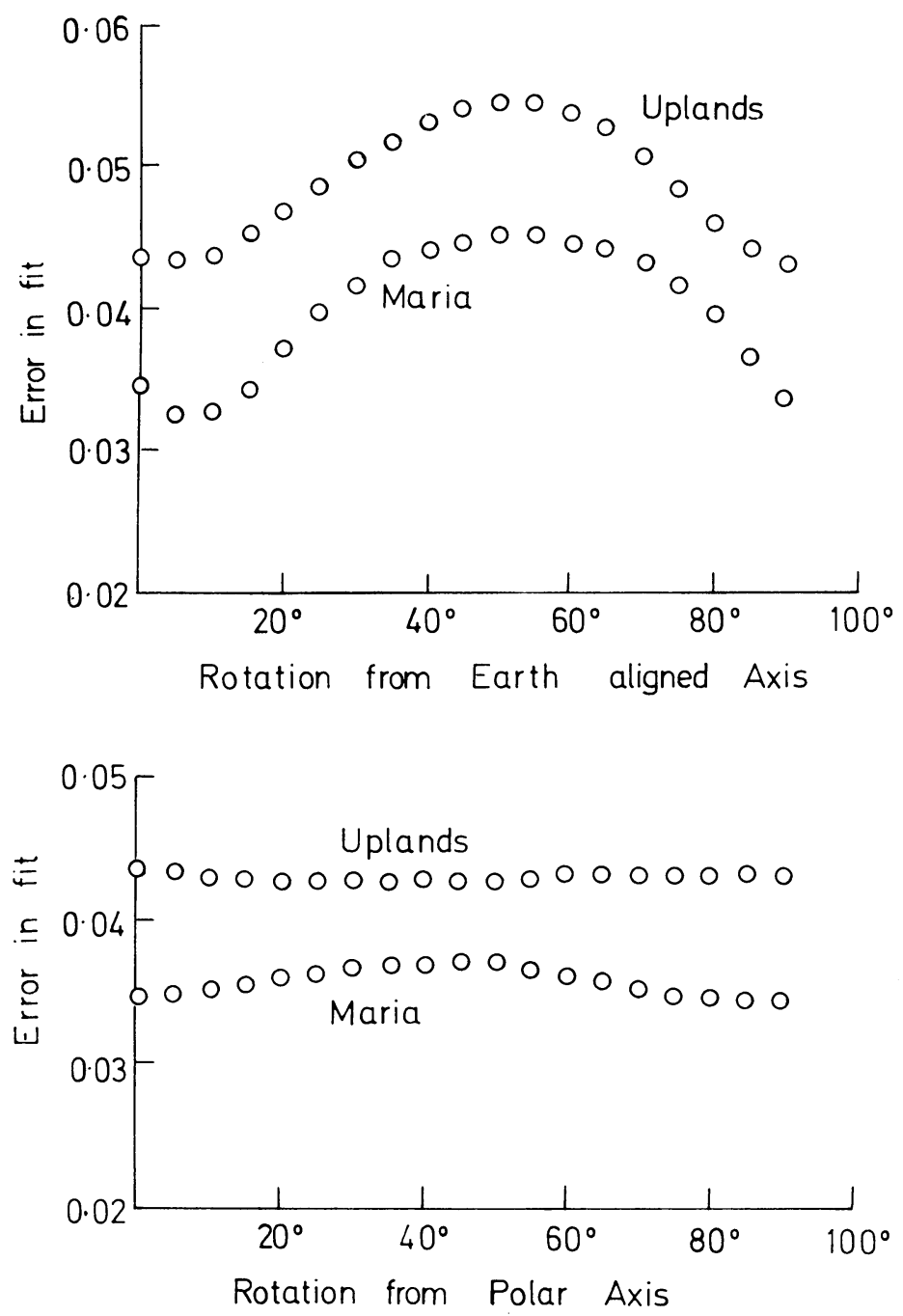

Fig. 2. Variation in the error of the fitted ellipsoids rotated about the polar axis and in the plane of the sky.

first when the ellipsoid is rotated about the polar axis $(z)$ and then in the plane of the sky. For the former there is a clear minimum when the $x$ axis coincides approximately with the Earth aligned axis whereas in the plane of the sky the variation is almost negligible.

The results of these analyses make it once more clearly apparent that the surface ellipticity greatly exceeds the dynamical ellipticity calculated from the differences in the moments of inertia $(C, A, B)$ about the polar axis $(z)$, the mean earthward direction $(x)$ (i.e. the direction of the Earth at nodal passage) and the equatorial axis in the plane of the sky $(y)$ respectively. 
As pointed out by Runcorn $(1962,1967)$ this is the key fact in understanding the cause of the non-hydrostatic condition of the Moon: it cannot simply result from the distortion of a uniform sphere in remote times. The interior of the Moon is not of uniform density: the density varies with angle so that the density below the centre of the disc is about $10^{-3}$ less than the density below its limb.

\section{Heights of the maria}

In their analysis, Runcorn and Shrubsall gave two maps on which is plotted the distribution of deviations of the points on the maria from the ellipsoid which best fits them and a similar one for the uplands. From the former it is clear that the surfaces of the circular maria which have the 'mascon' gravity anomalies above them, are systematically lower than those of the other maria by about $1 \mathrm{~km}$. This interesting result has been followed up on the Mills data by separating the maria into 'mascon

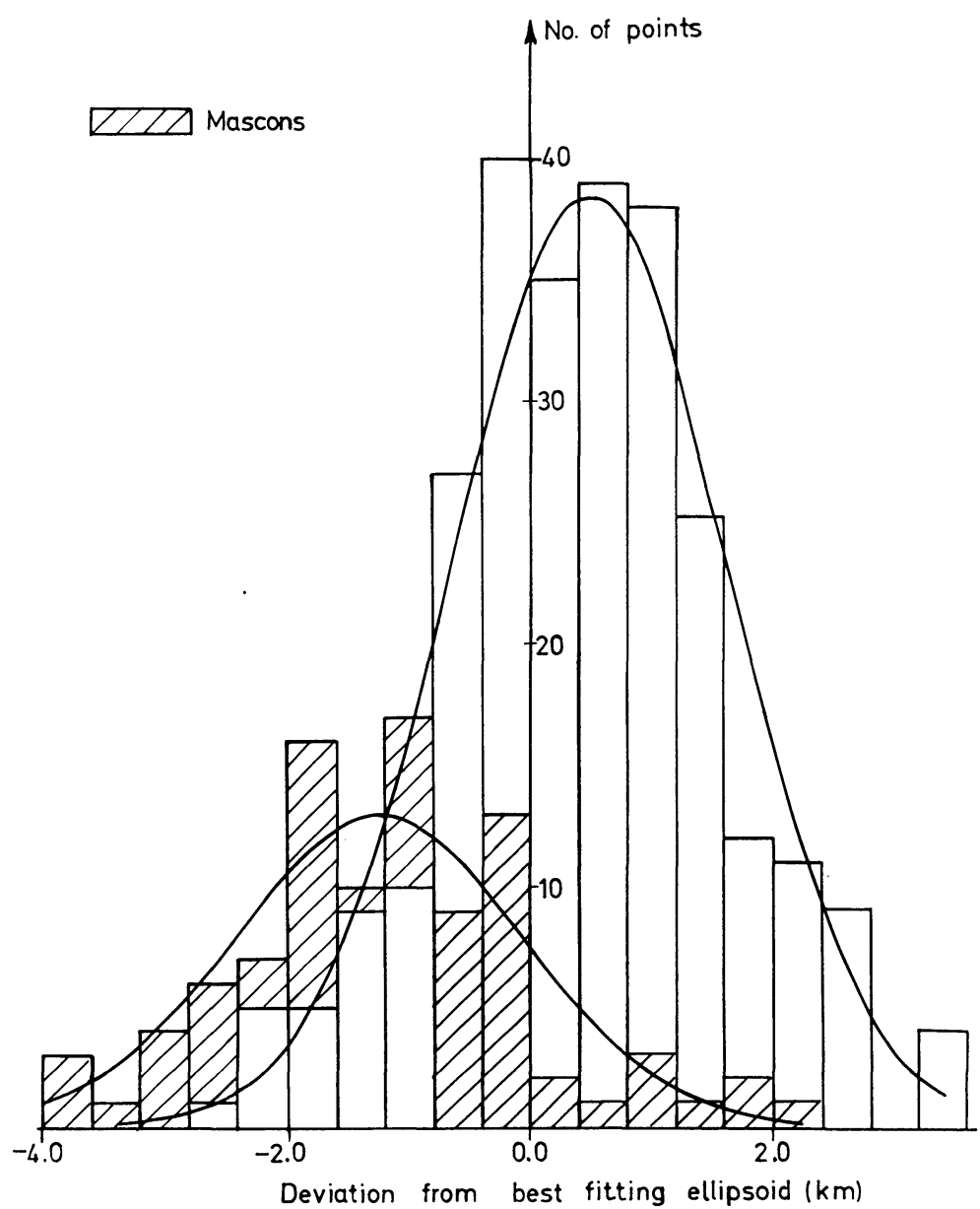

Fig. 3. Histograms showing the heights in the mascon maria and other maria. 
TABLE II

Axes of ellipsoids fitted to uplands, all maria, mascon maria and other maria

\begin{tabular}{lllllll} 
& $\begin{array}{l}\text { Semi-axis } \\
\text { toward Earth }\end{array}$ & $\begin{array}{l}\text { Equatorial } \\
\text { semi-axis in } \\
\text { plane of sky }\end{array}$ & $\begin{array}{l}\text { Polar } \\
\text { semi-axis }\end{array}$ & $\begin{array}{l}a_{x}- \\
\left(a_{y}+a_{z}\right) / 2\end{array}$ & $a_{y}-a_{z}$ & $\begin{array}{l}\text { Number } \\
\text { of points }\end{array}$ \\
Uplands & 1740.2 & 1737.3 & 1736.5 & 3.3 & 0.8 & 532 \\
All Maria & 1739.1 & 1736.0 & 1734.7 & 3.8 & 1.3 & 385 \\
Mascons & 1738.7 & 1734.5 & 1734.0 & 4.5 & 0.5 & 95 \\
Other Maria & 1739.3 & 1736.1 & 1735.2 & 3.7 & 0.9 & 290 \\
\hline
\end{tabular}

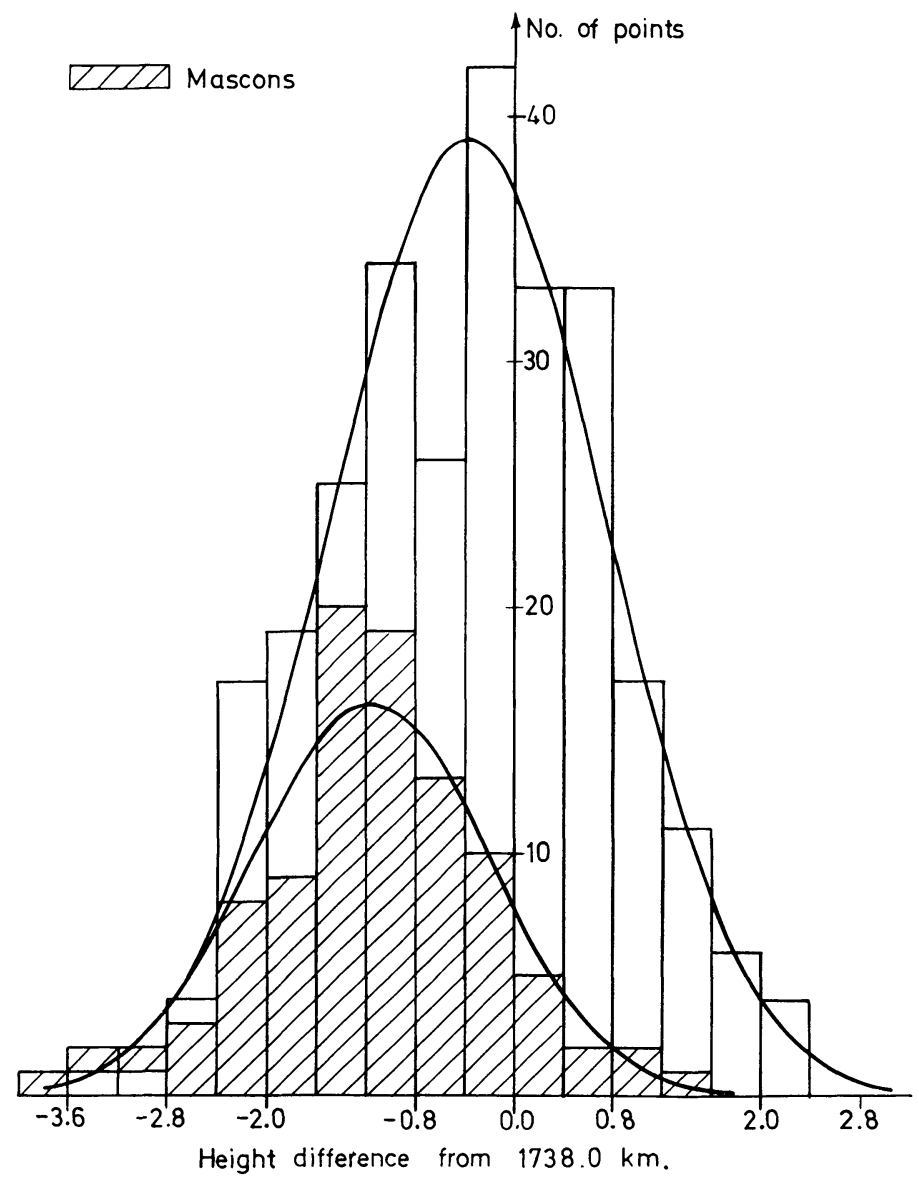

Fig. 4. Histograms showing the departures of the mascon maria and other maria from the best fitting ellipsoid to all maria. 


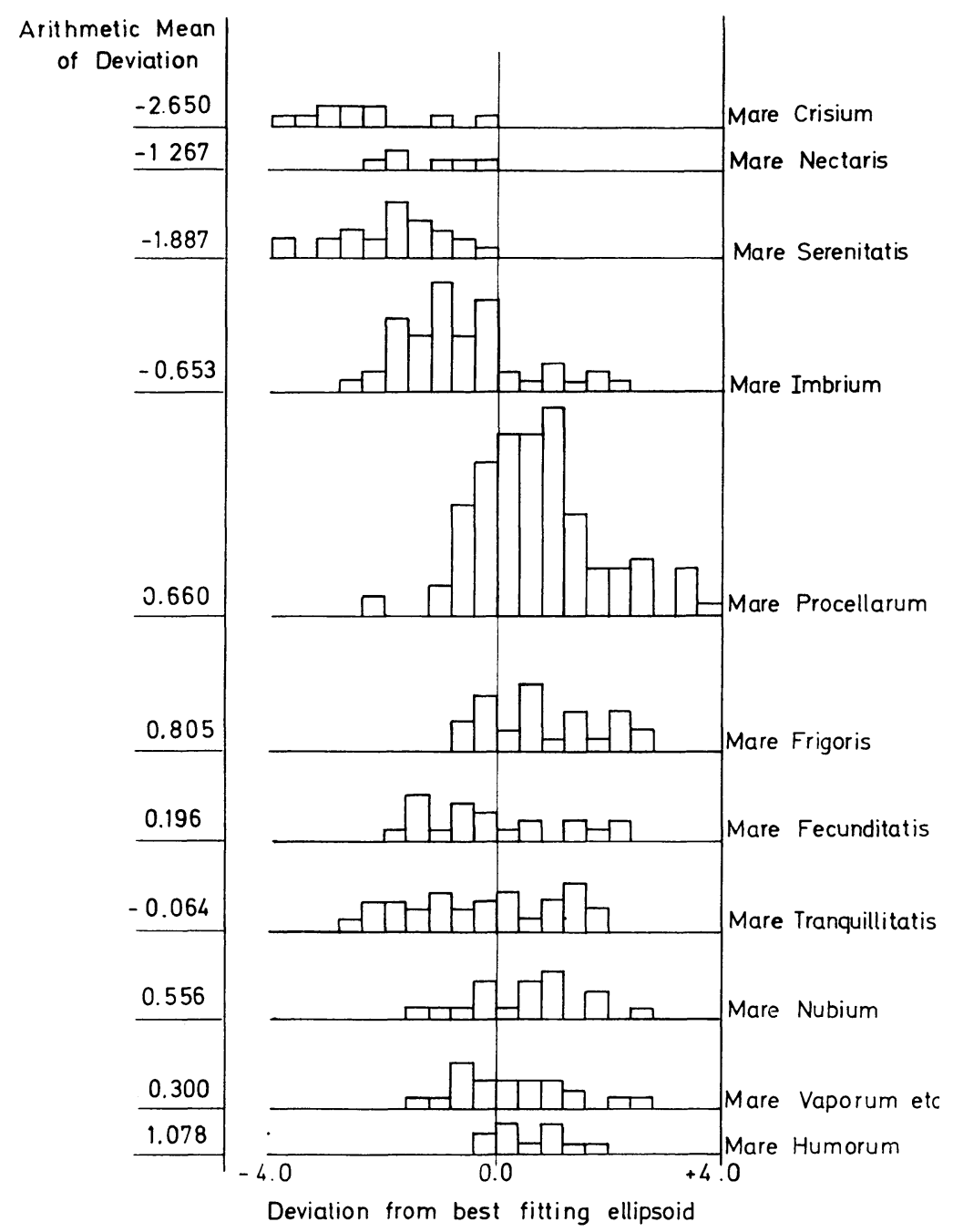

Fig. 5. Histograms showing the deviation of individual maria from the best fitting ellipsoid to all maria.

maria' and 'other maria' and fitting ellipsoidal surfaces to them. Table II shows the axis of the ellipsoids obtained. The 'shape' of the moon ellipsoid is maintained by these further surfaces.

To show further the systematic nature of this effect, Figure 3 is a histogram of the absolute heights of the two groups of maria and Figure 4 a histogram of the deviation of each group from the best fitting ellipsoid to all maria. The relatively small number of 'mascon maria' points available means that they do not contribute much when all maria are taken together.

Finally the deviation of each maria area from the best fitting ellipsoid to all maria 


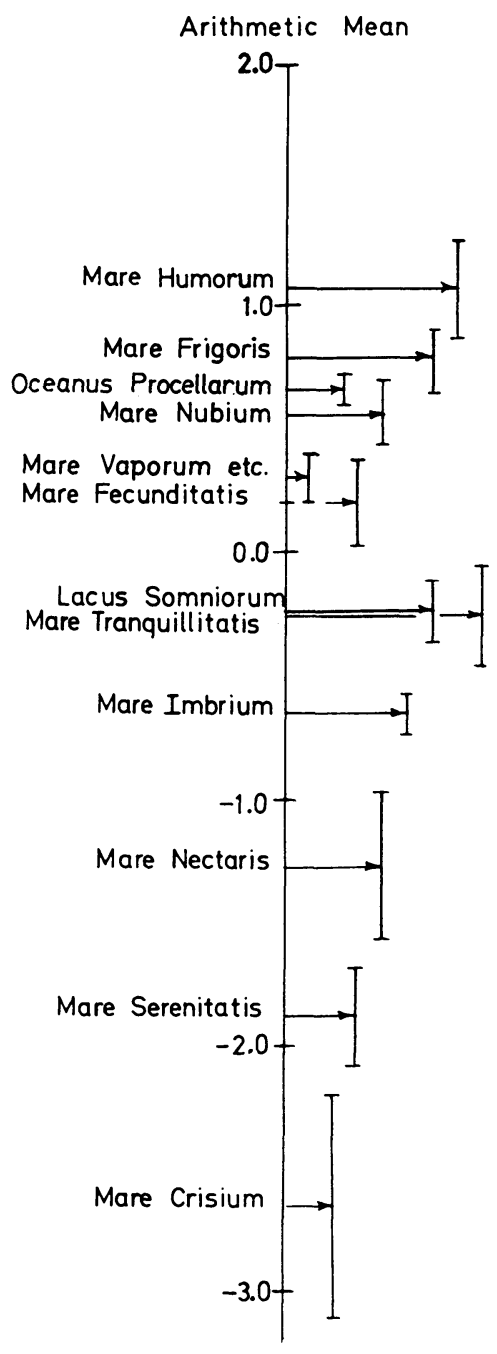

Fig. 6. Arithmetic mean of deviation of individual maria from best fitting ellipsoid showing standard deviation.

was considered separately. In Figure 5 the histogram of these deviations is plotted and in Figure 6 the arithmetic mean of the deviation and the standard mean deviation about this mean is shown. Mare Crisium emerges from this analysis as the lowest surface on the Moon.

\section{References}

Baldwin, R. B.: 1949, The Face of the Moon, Chicago University Press.

Baldwin, R. B.: 1963, The Measure of the Moon, Chicago University Press.

Goudas, C. L.: 1963, Icarus 2, 423.

Kopal, Z.: 1967, Proc. Roy. Soc. London 296, 254. 
Mills, G. A.: 1968, Icarus 8, 90.

Runcorn, S. K.: 1962, Nature, 195, 1150.

Runcorn, S. K.: 1967, Proc. Roy. Soc. London A296, 270.

Runcorn, S. K. and Gray, B. M.: 1967, in Mantles of the Earth and Terrestrial Planets, John Wiley, New York.

Runcorn, S. K. and Shrubsall, M. H.: 1968, Phys. Earth Planet. Interiors 1, 317. 